\title{
Modification of SRIM-Calculated Dose and Injected Ion Profiles Due to Sputtering, Injected Ion Buildup and Void Swelling
}

Jing Wang ${ }^{\text {a, } c^{*}}$, Mychailo B. Toloczko ${ }^{a}$, Nathan Bailey ${ }^{b}$, Frank A. Garner ${ }^{c}$, Jonathan Gigax ${ }^{c}$, Lin Shao $^{c}$

${ }^{a}$ Pacific Northwest National Laboratory, Richland, WA 99354, United States

${ }^{\mathrm{b}}$ University of California, Berkeley, CA 94720, United States

${ }^{\mathrm{c}}$ Texas A\&M University, College Station, TX 77843, United States

\begin{abstract}
In radiation effects on materials utilizing self-ion irradiations, it is necessary to calculate the local displacement damage level and ion injection profile because of the short distance that self-ions travel in a material and because of the strong variation of displacement rate with depth in a specimen. The most frequently used tool for this is the software package called Stopping and Range of Ions in Matter (SRIM). A SRIM-calculated depth-dependent dose level is usually determined under the implicit assumption that the target does not undergo any significant changes in volume during the process, in particular SRIM ignores the effect of sputtering, injected ions, and void swelling on the redistribution of the dose and injected ion profiles. This approach become increasingly invalid as the ion fluence reaches ever higher levels, especially for low energy ion irradiations. The original surface is not maintained due to sputterinduced erosion, while within the irradiated region of the specimen, injected ions are adding material, and if void swelling is occurring, it is creating empty space. An iterative mathematical treatment of SRIM outputs to produce corrected dose and injected ion profiles based on these phenomenon and without regard to diffusion is presented along with examples of differences between SRIM-calculated values and corrected values over a range of typical ion energies. The intent is to provide the reader with a convenient tool for more accurately calculating dose and injected ion profiles for heavy-ion irradiations.

*Corresponding author address: MS J4-55, 902 Battelle Blvd, Richland, WA 99354, United States. e-mail address: jing.wang@pnnl.gov; wang1943@tamu.edu
\end{abstract}

Key words: ion bombardment, SRIM, void swelling, sputtering, injected interstitial, dose assignment

1. Introduction

Microstructural changes that are induced by neutron irradiation in nuclear reactor core structural materials are a critical issue concerning safety and materials performance of both fast reactors and light water reactors (LWRs) [1,2]. Some current reactor concepts call for core structural components to withstand as much as 500-600 displacements per atom (dpa) of neutron irradiation [3, 4], while existing LWRs are being operated to lifetimes longer than originally planned, reaching perhaps $~ 200$ dpa in some locations 
over an 80 year operating lifetime [5]. Achieving such high dose levels in experimental studies on potentially more radiation-resistant materials via neutron irradiation is currently considered impractical to the lack of any available neutron irradiation sources that can achieve a useful dose in even a 5 year timeframe, especially in light of the wide range of new materials being considered for high dose structural materials. Therefore, if high dose is desired, it must be obtained using surrogate devices and techniques. Ion irradiation techniques at accelerated displacement rates are becoming widely employed again because high doses can be attained in days or even hours, rather than the decades it takes to generate the same damage levels with neutrons [6-9]. Depending on the specific accelerator design, ion irradiation is able to deliver as much as 50-600 dpa per day, many orders of magnitude higher than what can be achieved in reactors.

However, ion irradiations are not a direct substitute for neutron irradiations due to a number of factors usually described as neutron-atypical variables. Indeed, the best-known factor is that the dose rate is several orders of magnitude higher than that of a typical fast reactor core environment, thus having a strong effect not only on the point defect concentrations but on all temperature and rate-sensitive processes such as segregation, precipitation and void swelling. Other factors, such as the proximity and strong influence of the free surface, the ions injected by the ion irradiation [10], point defect imbalance effects [11] and depth-dependent compositional modifications arising from diffusion along the displacement rate gradients [12] are thought to affect the microstructural evolution of the target material to some degree.

Self-ion irradiations with energies $\leq 5 \mathrm{MeV}$ are now being conducted to very high (200-1000 dpa) displacement levels in order to study void swelling and microstructural stability in various structural alloys, especially for ferritic and ferritic-martensitic alloys and their oxide-dispersion-strengthened variants. The short range of self-ions and their energy-dependent damage deposition with depth result in depth-dependent dose and injected ion distributions. As discussed by Stoller et al. [13] the SRIM code [14] has become the de facto standard for calculations of damage dose and injected ion depth profiles. Stoller described a methodology for using SRIM to calculate dose levels for ion irradiations that are comparable to dose levels calculated for neutron irradiation experiments. The depth-dependent accumulated dose profile in ion irradiation is typically assumed to be proportional to the damage energy deposition that is calculated by SRIM. One of the simplifications of the SRIM-type Monte Carlo method is that it assumes that the target material layers are static with no feedback from sputtering or injected ions on the dose and injected ion profiles calculated from SRIM. However, sputtering removes atoms from the surface, effectively moving the interior volume closer to the incident surface while the injected ions increase the interior volume. These effects cause changes to the dose and injected ion profile not captured by SRIM and can be significant for higher doses, especially when the incident ion energy is low.

Sputtering removes surface atoms from the target surface when the energy transferred to them is greater than the surface binding energy. Sputtering also will act as a selective sink for removal of elements that diffuse more quickly to the surface, thus adding another layer of complexity to local composition variations resulting from ion irradiation. To the best of our knowledge, the effect of sputtering on dose and injected ion profiles has not been assessed in detail in the $\mathrm{MeV}$ energy range that is commonly used for studying irradiation effects on reactor core materials. Several previous sputtering studies using lower energy ion irradiations into metal targets were discussed in References [15-17]. In general, the sputtering effect is most significant at low ion energies $(\ll<1 \mathrm{MeV}$ for heavy ions like $\mathrm{Fe}, \mathrm{Ni}$, etc.) where sputtering 
yield peaks, and based on this, it was perceived as a secondary effect for higher energy heavy ion irradiations to moderate ion fluences [16]. For example, the surface erosion would be $\sim 12 \mathrm{~nm}$ if the sputtering yield is assumed to be 1 atom/ion in a Fe target irradiated to an ion fluence of $1 \times 10^{17}$ ion $/ \mathrm{cm}^{2}$ ( $\sim 100 \mathrm{dpa}$ at peak for $3.5 \mathrm{MeV} \mathrm{Fe}$ into $\mathrm{Fe}$ ). However, the effect becomes signficantly larger for high dose irradiation studies intended to reach peak damage levels 500-1000 dpa that require up to one order of magnitude higher ion fluence. Software packages, such as TRIDYN [18] and DYNA [19], have been developed to consider the surface sputtering and composition change during ion irradiation, but they are focused on fabrication of nanostructured semiconductors and surface modification at lower energies (approximately a few hundred keV) [19-24] and are not widely used for radiation damage studies.

In addition to sputtering, ion irradiations inject ions into the target material. There are two direct effects from such mass injection: the increase of the number of atoms and the change of chemical composition in the injected range. Johnston et al. [25-27] observed that the net thickness change arising from the opposing effects of sputtering and ion injection appeared to be negligible when measuring step height changes in the non-swelling regime of 304 and 316 stainless steels when irradiated with $5 \mathrm{MeV}$ Ni ions to fluence of $\sim 1 \times 10^{17}$ ions $/ \mathrm{cm}^{2}$ (corresponding to a calculated peak dose of $\sim 100 \mathrm{dpa}$ ). Johnston's technique had resolution limitations below $20 \mathrm{~nm}$ step-height, however.

As irradiations progress to ever higher doses, there is a need to evaluate the cumulative effects of sputtering, mass injection and void swelling on the dose and injected ion profiles. In contrast to Johnston's experimental evaluations, the work presented here assesses the impact of these processes using an iterative, integral summation of the sputtering rate and injected ion accumulation rate given by SRIM, providing a method that can be applied using typical SRIM outputs. The methodology presented here focuses only on modification of the dose and injected mass distributions and does not attempt to account for compositional variations with depth arising from diffusion.

Void swelling is a microstructural change that further complicates the dose and injected ion profiles due to the volume-averaged change in material density causing ions to penetrate to deeper depths compared to fully dense material $[27,28]$. Since considerable (double-digit) swelling has been observed in a number of high dose ion irradiations, swelling effects were incorporated into this methodology by adjusting dose and injected ion profiles based on a volume-averaged density change. It is important to note that both void swelling and injected ions contribute to volume increases in a very thin volume, but whereas the injected ions act only to increase the thickness, void swelling is a three dimensional volume change process. However, it is constrained laterally by the massive unirradiated substrate beyond the ion range. This constraint develops a membrane compressive stress state in the swelling layer with a shear vector normal to the incident ion surface, with the consequence that all mass flow from swelling moves only toward the specimen surface via irradiation creep [29-31]. In other words, the flow is one-dimensional, not threedimensional, as is characteristic of unconstrained volume increases.

Most published heavy ion irradiation studies do not address these effects on ion irradiation conditions. Some exceptions include a recent paper by Getto et al. that included the effect of void swelling on their dose calculations for $5 \mathrm{MeV}$ ion irradiation [32], and David et al. incorporated sputtering and injected interstitial contributions to step height measurements for void swelling [33]. Lack of widespread incorporation of these effects may be due to their unrecognized effect or may be due to a lack of a convenient tool to determine the details of these effects. This paper attempts to resolve both possible 
reasons for lack of usage by presenting a generalized approach to determine how sputtering, injected ions, and swelling affect dose and injected ion profiles.

\section{Calculation Method}

This method time-integrates the effects of sputtering and injected ion build-up in a one-dimensional model system and can be outlined by flow chart in Figure 1 that can be described in words as following:

1. The model system is spatially divided into $\mathrm{N}$ discrete bins. Quantities of interest, such as dose, injected ion concentration, and the total number of atoms are spatially associated with these bins.

2. Within a time-step, a certain flux of ions is injected into the model system. The changes in dose or injected ion concentration associated with these bins are directly applied from SRIMcalculated output for this number of ions.

3. Sputtering is then accounted for by removing atoms from the surface layer bin in the 1-D model system. Once the surface layer bin is empty, it is removed and the bin next to it becomes the new surface layer.

4. The injected ions are divided among the bins based on the SRIM-calculated probability distribution, and the total number of atoms in those bins is increased (thus the size of the bin changes). Both the dose and the injected ion concentration profiles are then corrected for the addition of injected ions.

5. Steps 2 to 4 are repeated until total ion fluence reaches the target value. Note that the processing sequence of step 3 and 4 is switched after every execution of the loop.

The details of the calculation are explained here. While ion fluence is expressed in ions (or atoms) per unit area (commonly, ions $/ \mathrm{cm}^{2}$ or atoms $/ \mathrm{cm}^{2}$ ), it is more convenient to use mass depth (ions $/ \mathrm{cm}^{2}$ ) as the unit for addressing the position of each bin in the 1-D system [27]. Mass depth is the multiplication of physical depth (e.g. in $\mathrm{cm}$ ) and atomic density (e.g. atoms $/ \mathrm{cm}^{3}$ ) in a cumulative manner. For example, a physical depth of $1 \mathrm{~cm}$ is equivalent to $8.48 \times 10^{22}$ atoms $/ \mathrm{cm}^{2}$ in mass depth, assuming the atomic density of pure $\mathrm{Fe}$ is $8.48 \times 10^{22}$ atoms $/ \mathrm{cm}^{3}$. This procedure automatically incorporates the number of atoms in the system for the calculation. All computations are carried out based on mass depth and then converted for display to physical depth based on the atomic density of each bin after the last iteration.

For Step 2, the process is expressed as simple addition of values stored at mass depth $x$ and SRIMevaluated increments at that position by

$$
\begin{gathered}
D P A(x, t+\Delta t)=\operatorname{DPA}(x, t)+\operatorname{DPA}(x, \Delta t) \\
I N J(x, t+\Delta t)=\operatorname{INJ}(x, t)+\operatorname{INJ}(x, \Delta t)
\end{gathered}
$$

where

- $\quad x$ is the mass depth associated with the bin

- $\quad t$ is the time of ion irradiation and $\Delta t$ is the length of time step

- $\operatorname{DPA}(x, t)$ and $I N J(x, t)$ are the dose and injected ion concentration at depth $x$ and time $t$. The injected ions are measured in terms of mass depth (areal density, atoms $/ \mathrm{cm}^{2}$ ). 
The surface sputtering in Step 3 effectively removes atoms from the surface bin. Considering the original thickness of the surface bin in mass depth to be $x_{0}$, the new surface layer will be $x_{0}-\Delta x_{s p t}$ thick after sputtering of atoms equivalent to $\Delta x_{s p t}$ in mass depth. The entire $x$ coordinate is thus shifted by the amount $\Delta x_{s p t}$ to make the new surface start at 0 for convenience. The process is expressed by

$$
\begin{aligned}
& D P A\left(x_{\text {new }}, t+\Delta t\right)=D P A\left(x-\Delta x_{\text {spt }}, t+\Delta t\right) \\
& I N J\left(x_{\text {new }}, t+\Delta t\right)=I N J\left(x-\Delta x_{\text {spt }}, t+\Delta t\right)
\end{aligned}
$$

where

- $\quad x_{\text {new }}$ is the new coordinate of mass depth for the next iteration

- $\Delta x_{s p t}$ is the mass depth corresponding to the amount of sputtered materials, which is equal to the multiplication of sputtering yield and injected ion flux per time step.

The injection of ions into the target could have two effects: an increase in material thickness and a change in chemical composition. The injected ions eventually come to rest and increase the atom numbers within bins. Assuming atomic density remains the same, the thicknesses of those bins change as described by

$$
x_{i}(t+\Delta t)-x_{i-1}(t+\Delta t)=x_{i}(t)-x_{i-1}(t)+I N J\left(x_{i}, \Delta t\right), \mathrm{i}=1,2,3 \ldots \mathrm{N}-1
$$

where $i$ is the bin index and $x_{i}-x_{i^{-}}$is the thickness of the $i^{\text {th }}$ bin in terms of mass depth. Since dose and the concentration of injected ions are quantities that rely on the total number of atoms in each bin, their values need to be re-scaled by

$$
\begin{gathered}
D P A_{\text {new }}\left(x_{i}, t+\Delta t\right)=\frac{x_{i}(t)-x_{i-1}(t)}{x_{i}(t+\Delta t)-x_{i-1}(t+\Delta t)} D P A\left(x_{i}, t+\Delta t\right), \mathrm{i}=1,2,3 \ldots \mathrm{N}-1 \\
C\left(x_{i}, t+\Delta t\right)=\frac{\operatorname{INJ}\left(x_{i}, t+\Delta t\right)}{n_{0}+I N J\left(x_{i}, t+\Delta t\right)}, \mathrm{i}=1,2,3 \ldots \mathrm{N}-1
\end{gathered}
$$

where $n_{0}$ is number of atoms in each bin at the beginning, and $C\left(x_{i}, t\right)$ is the concentration of injected ions in the $i^{\text {th }}$ bin.

Finally, mass depth is converted to physical depth by

$$
d_{i}(t)=\frac{x_{i}(t)}{\rho_{i}(t)}, i=1,2,3 \ldots \mathrm{N}-1
$$

where $\rho_{i}(t)$ is the atomic density of the $i^{\text {th }}$ bin. Atomic density is assumed to be constant for the special case of no void swelling. The accuracy of the numerical integration process was analyzed by comparing the output from a range of time steps as shown in Figures $2 \mathrm{a}$ ) and b) for the $1.8 \mathrm{MeV}$ ion case. A timestep of sufficient granularity was selected to be within a range of values where the results were nearly invariant with increases or decreases in the size of the time step while minimizing computational time. In this case a fluence step of $4 \times 10^{17}$ was sufficient to reach invariance. The effect of switching the order of Step 2 (ion injection) and Step 3 (sputtering) was investigated and found to have a negligible difference on the outcome for sufficiently small time steps. 
When void swelling occurs, the injected ions travel farther into the material relative to the incident surface. Odette et al. [28] mathematically analyzed the effects of voids on energy deposition and stopping ranges of particles in materials. His study reported an extension of the average projected range, and that straggling of particles could be significant at the end of the range. It was found that a mass density correction could adequately account for the presence of voids, except at end of range (due to straggling). In the method presented here, void swelling can be accounted for in a similar manner by treating swelling as a decrease in local atomic density

$$
\rho_{i}(t)=\frac{\rho_{i}\left(t_{0}\right)}{1+S_{i}(t)}, \mathrm{i}=1,2,3 \ldots \mathrm{N}-1
$$

where $S_{i}(t)$ is the swelling of the $i^{t h}$ bin, $\rho_{i}\left(t_{0}\right)$ is the density of a non-swollen bin. This methodology does not take into account straggling effects at the end of range.

The cumulative dose profile and injected ion distribution as a function of mass depth can be obtained by iterating through Equations (1)-(6) over time until the target dose is achieved. All results based on mass depth can then be converted to physical depth using Equations (8) and (9).

Historically, ion irradiations have been performed with various ion species ( $\mathrm{H}, \mathrm{C}, \mathrm{Fe}, \mathrm{Ni}, \mathrm{Ti}, \mathrm{Ar}, \mathrm{Au}, \mathrm{Xe}$, etc.) that span a wide range of energies. High dose ion irradiations are most often performed on Fe-based austenitic and ferritic-martensitic alloys using self-ion irradiation of the most abundant compositional elements, namely $\mathrm{Ni}, \mathrm{Cr}, \mathrm{Fe}$, although many of the earliest swelling-oriented studies on austenitic steels used $500 \mathrm{keV}$ Ni or $\mathrm{Cu}$ ions [34-36].

Due to the current popularity of iron based ferritic alloys for core structural materials, we employ a pure Fe target irradiated with Fe ions at energies of $500 \mathrm{keV}, 1.8 \mathrm{MeV}, 3.5 \mathrm{MeV}$ and $5 \mathrm{MeV}$ in order to show examples of the range of significance of these effects. In this analysis, the ion flux is not relevant and we focus only on the ion fluence. The dose range studied was very large, approaching $1000 \mathrm{dpa}$ at the peak damage depth, depending on the ion energy used. While at first glance this very high dose level appears to be out of the realm of technological interest, it is important to recognize that modern ion heavy ion irradiation studies focus on the effect of irradiation at a depth that is before the peak damage depth, typically with the dose at this depth being approximately half the value at the peak damage depth.

Sputtering yield per incident ion as determined by SRIM is related to the ion species, incident angle, ion energy, and surface binding energy. Experimentally, however, surface sputtering has been reported to be sensitive to ion beam flux, surface roughness and chamber vacuum level as well [24, 37, 38]. Grain orientation is also known to affect sputtering [30, 31]. Extensive reviews of sputtering process are available elsewhere [39, 40]. For simplicity, in this study SRIM-estimated sputtering yields were calculated with the SRIM-suggested Fe surface binding energy (SBE) of $4.34 \mathrm{eV}$, using the "monolayer collision step/surface sputtering" option, the displacement threshold energy set to $40 \mathrm{eV}$, and the lattice binding energy set to $0 \mathrm{eV}$ [41]. Among all SRIM input parameters, the SBE of the target material most significantly affects the resulting sputtering yield. The SBE is the minimum energy normal to the target surface required for an atom to escape. Due to lack of experimental data on SBE, the default value in SRIM and several other common Monte Carlo programs, such as TRIDYN, is to adopt the heat of sublimation as an estimate. Theoretical estimations of SBE for Fe are reported to vary from 2.3 to $5.1 \mathrm{eV}$ in the literature $[42,43]$. Since the commonly used heat of sublimation (as SBE) is likely obtained at 
thermodynamic standard conditions for temperature and pressure (273 K and $100 \mathrm{kPa})$, it adds another level of uncertainty when applied to estimate sputtering yield in high temperature ion irradiations where thermal vibrations of surface atoms could potentially increase emission cross-section, especially with a low energy cascade collision [44]. Therefore, there is substantial uncertainty in the sputtering yields presented here, and more accurate SBE values would be of value for assessing the effects of sputtering as described here.

The treatment just described assumed a simplified, static model where diffusion and defect reactions were ignored as is the case with SRIM. However, diffusional processes may play a substantial interactive role in reshaping the injected ion profile. The free surface could also play a role by acting as a strong defect sink.

\section{Results and discussion}

Table 1 lists the SRIM-calculated sputtering yield for Fe ions bombarding pure iron with energies ranging from 0.5 to $5.0 \mathrm{MeV}$. At low energies, the sputtering yield per incident ion is greater by a factor of 3-4 than that calculated for higher energies. Thus, it is expected that the greatest sputter-injection correction and the greatest effect of time-step size will be at lowest ion energy.

Examples of the effect of sputtering and injected ion are first presented in absence of any swelling. Figure 3 compares the uncorrected and corrected dose and injected ion profiles for $500 \mathrm{keV}$ irradiations. Note that the origin of the $\mathrm{x}$-axis at $0 \mathrm{~nm}$ always represents the current surface of the target material. The corrected dose and injected ion profiles agree with the SRIM-calculated curves at the low fluence of $1 \times 10^{16}$ ions $/ \mathrm{cm}^{2}$ ( 16 dpa at the peak, based on SRIM) as shown in Figures 3a) and b). At this fluence, only $\sim 4 \mathrm{~nm}$ of surface material has been sputtered away, while the average injected ion range is $\sim 175 \mathrm{~nm}$. The disparity between the corrected profiles and the SRIM results progressively increases as fluence is increased as depicted in Figures 3c) and d). Both the sputtering and injected ions act to broaden the distribution curves. Sputtering of material from the surface causes the positions of the integrated damage and injected ion peaks to move toward the surface, while at the same time broadening these two curves through the depth. This latter effect causes a reduction in height of the peaks relative to the SRIMcalculated curves. At $5 \times 10^{17}$ ions $/ \mathrm{cm}^{2}$ ( 770 dpa at the peak based on SRIM), the shape of the dose distribution has drastically changed, and the corrected ion-incident surface has an injected ion concentration of $\sim 17$ at $\%$, as shown in Figures 3c) and d). In effect, the number of injected ions is now becoming a significant fraction of the sputtered ions.

Figure 4 presents the uncorrected and corrected dose and injected ion distributions for $1.8 \mathrm{MeV}, 3.5 \mathrm{MeV}$ and 5.0 MeV ions. Since sputtering yields are lower and ion ranges are longer at higher energies, the effects of sputtering and ion injection are not as strong as those shown in Figure $3(500 \mathrm{keV})$, and are decreasing with increasing ion energy. At the low fluence of $1 \times 10^{17}$ in Figures $4 a$ ) and b), the iteratively calculated distributions are very close to the SRIM predictions.

At the higher fluences shown in Figures 4c), d), e) and f), however, the corrected peaks are progressively shifted toward the surface relative to the SRIM curves and are reduced in height just as was observed for $500 \mathrm{keV}$ ions in Figures 3c) and d). The peak broadening effect seen in Figure 4 is is not as strong at higher ion energies compared to Figure 3 . This can be attributed to the lower sputtering yield at higher ion energies, causing less peak shift toward the surface, and the broadening contribution from the injected 
ions is not as strong because the distributions themselves are wider at higher energies. For example, irradiation of $5 \times 10^{17}$ ions $/ \mathrm{cm}^{2}$ would inject about $59 \mathrm{~nm}$ of atoms into the target; however, the relative broadening from this amount of material is not significant for $5.0 \mathrm{MeV}$ where the dose and injected ion profiles are both more than $1 \mu \mathrm{m}$ wide. Figures 3 and 4 clearly illustrate that sputtering and injected ion effects can result in substantial changes to the predicted profiles at lower accelerating energies.

The ion beam energy affects both the sputtering rate and the ion stopping depth. The amount of material removed and the average ion range, $R_{p}$, for these three ion energies are listed in Table 1 for an ion fluence of $5 \times 10^{17}$ ions $/ \mathrm{cm}^{2}$. Figure $4 \mathrm{e}$ ) indicates that the region in front of the damage and deposition peaks, where microstructural observations are now routinely performed to avoid effects of injected interstitial atoms, will have a higher dose and injected ion concentration than predicted by SRIM. For instance, for $1.8 \mathrm{MeV}$ ions at a depth of $200 \mathrm{~nm}$, SRIM predicts $700 \mathrm{dpa}$, but the corrected calculation predicts 850 dpa.

Increasing ion energy can alleviate this dose modification effect. A similar outcome is observed for the distributions of injected ions. While these results show the injected ion profile being redistributed, it is important to understand that this is an evolutionary process and that the SRIM-predicted profile represents the location where the injected ions will initially come to rest. The cumulative effects of sputtering and injected ions then progressively alter the profiles by moving the entire volume of material.

The aforementioned results have not accounted for swelling. The influence of swelling on the corrected dose and injected ion profiles at a given fluence can be accomplished by applying the observed swelling profile (as observed in cross section) at that fluence to Equation (9) and then converting mass depth to physical depth using Equation (8). This is conveniently accomplished by using the bins set up for calculating the corrected dose and injected ion profiles.

An example of the effect swelling on the dose and injected ion profiles is presented here. Since it is not the purpose of this paper to discuss the origin and evolution of swelling with respect to irradiation, a Gaussian type swelling profile has been assumed. A Gaussian is a reasonable approximation to swelling profiles observed in some ion irradiation experiments $[3,7,11,45]$ where swelling was suppressed from injected ions at the back of the ion range and from free-surface effects at the front of the range.

To illustrate the effects of different swelling levels, peak swelling values of $\sim 25 \%$ and $\sim 50 \%$ were selected for an ion fluence of $6.5 \times 10^{17}$ ions $/ \mathrm{cm}^{2}$, which is equivalent to $500 \mathrm{dpa}$ at 200-300 nm estimated by SRIM for $1.8 \mathrm{MeV}$ Fe. Values of this magnitude have been observed in $1.8 \mathrm{MeV} \mathrm{Cr}$ irradiations in ferritic and austenitic alloys $[46,47]$. The peak positions of swelling profile were fixed to a depth corresponding to the same mass depth in both test cases. Figure 5a) and c) shows that within the swelling region, the swelling-corrected dose, as indicated by "+Swelling Corrected" curves was lower than the corrected dose that accounts only for sputtering and injected ions. At the depth of peak swelling in Figure 5a), the SRIM dose is $\sim 510 \mathrm{dpa}$, while the corrected value that accounts for sputtering and ion injection is $590 \mathrm{dpa}$, and with the swelling correction added it is $\sim 570 \mathrm{dpa}$. Another effect from swelling is that injected ion distributions are shifted away from the surface as shown in Figure 5b) and d). The resulting adjustments to the profiles may be of interest when studying suppression of void nucleation from injected ions since the ion range increases as swelling increases. 
Application of this swelling correction to a real data set is provided in Figure 6 that shows a comparison dose and injected ion profiles as calculated by SRIM alone and determined by the methods presented here for a Russian ferritic-martensitic steel, EK-181, irradiated by $3.5 \mathrm{MeV} \mathrm{Fe}^{2+}$ to 400 peak dpa at $475{ }^{\circ} \mathrm{C}$ [48]. The swelling profile was obtained in TEM cross-section specimens prepared by a focused ion beam lift-out technique. Both the dose and injected ions distributions were extended deeper into the target materials and their values around the peak swelling depth were slightly lower. The lowered dpa value, in Figure 6a) may have subtle effects when evaluating swelling-dpa relationship, for example the swelling/dpa ratio would be $\sim 10 \%$ higher using swelling corrected values than the SRIM-estimated value.

\section{Summary}

The SRIM code is the currently accepted tool for calculation of displacement dose and injected ion profiles for irradiation effects studies using ion bombardment. It was shown here that the assumption of a static target not influenced by sputtering or injected ions may be insufficient for calculating accurate dose and injected ion profiles at higher doses with lower ion energies. The extent of this effect at higher ion energies may not be fully understood until more accurate SBE values are obtained. A simple iterative mathematical treatment was presented that allows obtaining the more accurate dose and injected atom profiles from SRIM outputs. For completeness, the effect of swelling was also included in this study. For materials that swell readily, care must be taken when determining the local dose since void swelling effectively expands and broadens the damage profile even when sputtering is low. The results suggest that the impact of swelling on the dose profile in the region prior to the swelling peak is relatively small. Interestingly, the impact of void swelling on the dose and injection profiles runs counter to the effect of sputtering effect, tending to shift them away from the surface compared to sputtering moving the profiles towards the surface.

Acknowledgements:

This research was funded by the Fuel Cycle R\&D Program Core Materials research area sponsored by the U.S. Department of Energy, Office of Nuclear Energy. Pacific Northwest National Laboratory is operated for the U.S. Department of Energy by Battelle Memorial Institute under Contract DE-AC0676RLO 1830.

\section{References:}

[1] F.A. Garner, Radiation Damage in Austenitic Steels, in: R.J. Konings (Ed.), Comprehensive Nulcear Materials, Elsevier2012, pp. 33-95.

[2] F.A. Garner, Void swelling and irradiation creep in light water reactor (LWR) environments, in: P.G. Tipping (Ed.), Understanding and Mitigating Aging in Nulear Power Plants, Woodhouse Publishing2010, pp. 308-356.

[3] G.S. Was, Challenges to the use of ion irradiation for emulating reactor irradiation, Journal of Materials Research 30(09) (2015) 1158-1182.

[4] M.B. Toloczko, F.A. Garner, C.R. Eiholzer, Irradiation creep and swelling of the US fusion heats of HT9 and 9Cr-1Mo to 208 dpa at 400 C, Journal of nuclear materials 212 (1994) 604-607.

[5] E.A. Kenik, J.T. Busby, Radiation-induced degradation of stainless steel light water reactor internals, Materials Science and Engineering: R: Reports 73(7) (2012) 67-83.

[6] G. Odette, M. Alinger, B. Wirth, Recent developments in irradiation-resistant steels, Annu. Rev. Mater. Res. 38 (2008) 471-503. 
[7] X. Wang, A.M. Monterrosa, F. Zhang, H. Huang, Q. Yan, Z. Jiao, G.S. Was, L. Wang, Void swelling in high dose ion-irradiated reduced activation ferritic-martensitic steels, Journal of Nuclear Materials 462 (2015) 119-125.

[8] C. Wei, A. Aitkaliyeva, M. Martin, D. Chen, L. Shao, Microstructural changes of T-91 alloy irradiated by Fe self ions to ultrahigh displacement ratios, Nuclear Instruments and Methods in Physics Research Section B: Beam Interactions with Materials and Atoms 307 (2013) 181-184.

[9] M.B. Toloczko, F. Garner, V. Voyevodin, V. Bryk, O. Borodin, V. Mel'nychenko, A. Kalchenko, Ioninduced swelling of ODS ferritic alloy MA957 tubing to 500dpa, Journal of Nuclear Materials 453(1) (2014) 323-333.

[10] F.A. Garner, Impact of the injected interstitial on the correlation of charged particle and neutroninduced radiation damage, Journal of Nuclear Materials 117 (1983) 177-197.

[11] L. Shao, C.-C. Wei, J. Gigax, A. Aitkaliyeva, D. Chen, B.H. Sencer, F.A. Garner, Effect of defect imbalance on void swelling distributions produced in pure iron irradiated with $3.5 \mathrm{MeV}$ self-ions, Journal of Nuclear Materials 453(1) (2014) 176-181.

[12] V.A. Pechenkin, A.D. Chernova, F.A. Garner, Modeling of Local Changes in Alloy Composition along the Projected Range under High Dose Irradiation, Accelerator Application, Bruges, Belgium, 2013.

[13] R.E. Stoller, M.B. Toloczko, G.S. Was, A.G. Certain, S. Dwaraknath, F.A. Garner, On the use of SRIM for computing radiation damage exposure, Nuclear Instruments and Methods in Physics Research Section B: Beam Interactions with Materials and Atoms 310 (2013) 75-80.

[14] J.F. Ziegler, J.P. Biersack, The stopping and range of ions in matter, Springer 1985.

[15] G.S. Was, Ion beam modification of metals: compositional and microstructural changes, Progress in Surface Science 32(3-4) (1989) 211-332.

[16] D.J. Mazey, Fundamental aspects of high-energy ion-beam simulation techniques and their relevance to fusion materials studies, Journal of Nuclear Materials 174(2) (1990) 196-209.

[17] N. Lam, H. Wiedersich, Bombardment-induced segregation and redistribution, Nuclear Instruments and Methods in Physics Research Section B: Beam Interactions with Materials and Atoms 18(1) (1986) 471-485.

[18] W. Möller, W. Eckstein, J.P. Biersack, Tridyn-binary collision simulation of atomic collisions and dynamic composition changes in solids, computer physics communications 51(3) (1988) 355-368.

[19] A.L. Stepanov, V.A. Zhikharev, I.B. Khaibullin, Depth profiles of metal ions implanted in dielectrics at low energies, Physics of the Solid State 43(4) (2001) 766-771.

[20] A. Johannes, H. Holland-Moritz, C. Ronning, Ion beam irradiation of nanostructures: sputtering, dopant incorporation, and dynamic annealing, Semiconductor Science and Technology 30(3) (2015) 033001.

[21] D.E. Hole, A.L. Stepanov, P.D. Townsend, Dependence of optical properties of implanted silver nanoparticles in float glass on substrate temperature, Nuclear Instruments and Methods in Physics Research Section B: Beam Interactions with Materials and Atoms 148(1) (1999) 1054-1058.

[22] S.T. Picraux, Ion implantation in metals, Annual Review of Materials Science 14(1) (1984) 335-372.

[23] W. Möller, TRI3DYN-Collisional computer simulation of the dynamic evolution of 3-dimensional nanostructures under ion irradiation, Nuclear Instruments and Methods in Physics Research Section B: Beam Interactions with Materials and Atoms 322 (2014) 23-33.

[24] M.A. El Khakani, H. Jaffrezic, G. Marest, N. Moncoffre, J. Tousset, Sputtering yield and residual vacuum influence during titanium implantation into iron, Nuclear Instruments and Methods in Physics Research Section B: Beam Interactions with Materials and Atoms 50(1) (1990) 406-409.

[25] W.G. Johnston, J.H. Rosolowski, A.M. Turkalo, K.D. Challenger, Surface observations of nickel ion-bombarded stainless steels, Scripta Metallurgica 6(10) (1972) 999-1005.

[26] W.G. Johnston, J.H. Rosolowski, A.M. Turkalo, T. Lauritzen, A direct measurement of gross swelling in nickel-ion-bombarded stainless steel, Journal of Nuclear Materials 46(3) (1973) 273-280.

[27] W.G. Johnston, J.H. Rosolowski, A.M. Turkalo, T. Lauritzen, Nickel ion bombardment of type 304 stainless steel: comparison with fast reactor swelling data, Journal of Nuclear Materials 47(2) (1973) 155167. 
[28] G.R. Odette, D.M. Schwartz, A.J. Ardell, Particle range and energy deposition in materials containing voids, Radiation Effects 22(4) (1974) 217-223.

[29] F.A. Garner, G.L. Wire, E.R. Gilbert, Stress Effects in Ion Bombardment Experiments, in: J.S. Watson, F.W. Wiffen (Eds.) Radiation Effects and Tritium Technology, CONF-750989, 1976, pp. I-474.

[30] W.G. Wolfer, F.A. Garner, Swelling-induced stresses in ion-bombarded surfaces: effect of crystalline orientation, Journal of Nuclear Materials 85 (1979) 583-589.

[31] F.A. Garner, D.S. Gelles, Irradiation creep mechanisms: an experimental perspective, Journal of Nuclear Materials 159 (1988) 286-309.

[32] E. Getto, K. Sun, S. Taller, A.M. Monterrosa, Z. Jiao, G.S. Was, Methodology for determining void swelling at very high damage under ion irradiation, Journal of Nuclear Materials 477 (2016) 273-279.

[33] C. David, B.K. Panigrahi, S. Balaji, A.K. Balamurugan, K.G.M. Nair, G. Amarendra, C.S. Sundar, B. Raj, A study of the effect of titanium on the void swelling behavior of D9 steels by ion beam simulation, Journal of Nuclear Materials 383(1) (2008) 132-136.

[34] L. Glowinski, C. Fiche, M. Lott, Etude de la formation des cavités dans le cuivre irradie aux ions cuivre de $500 \mathrm{keV}$, Journal of Nuclear Materials 47(3) (1973) 295-310.

[35] P. Regnier, L.D. Glowinski, Effet du flux instantane sur la formation des cavites dans le nickel irradie aux ions Ni+ de $500 \mathrm{keV}$, Journal of Nuclear Materials 57(2) (1975) 243-245.

[36] L.D. Glowinski, C. Fiche, Etude de la formation des cavites d'irradiation dans le cuivre II-irradiation aux ions cuivre de $500 \mathrm{keV}$ - effet de la dose, Journal of Nuclear Materials 61(1) (1976) 22-28.

[37] O. Almen, G. Bruce, Sputtering experiments in the high energy region, Nuclear Instruments and Methods 11 (1961) 279-289.

[38] Y.G. Li, Y. Yang, M.P. Short, Z.J. Ding, Z. Zeng, J. Li, IM3D: A parallel Monte Carlo code for efficient simulations of primary radiation displacements and damage in 3D geometry, Scientific reports 5 (2015).

[39] P. Sigmund, Theory of sputtering. I. Sputtering yield of amorphous and polycrystalline targets, Physical review 184(2) (1969) 383.

[40] P. Sigmund, Recollections of fifty years with sputtering, Thin Solid Films 520(19) (2012) 60316049.

[41] H. Hofsäss, K. Zhang, A. Mutzke, Simulation of ion beam sputtering with SDTrimSP, TRIDYN and SRIM, Applied Surface Science 310 (2014) 134-141.

[42] Y. Kudriavtsev, A. Villegas, A. Godines, R. Asomoza, Calculation of the surface binding energy for ion sputtered particles, Applied surface science 239(3) (2005) 273-278.

[43] M.A. Karolewski, Tight-binding potentials for sputtering simulations with fcc and bcc metals, Radiation effects and defects in solids 153(3) (2001) 239-255.

[44] Q. Yan, J. Wang, D. Chen, J. Gigax, L. Shao, Displacement cross sections of electron irradiated graphene and carbon nanotubes, Nuclear Instruments and Methods in Physics Research Section B: Beam Interactions with Materials and Atoms 350 (2015) 20-25.

[45] M.P. Short, S. Yip, Materials aging at the mesoscale: Kinetics of thermal, stress, radiation activations, Current Opinion in Solid State and Materials Science (2015).

[46] A.S. Kalchenko, V.V. Bryk, N.P. Lazarev, I.M. Neklyudov, V.N. Voyevodin, F.A. Garner, Prediction of swelling of $18 \mathrm{Cr} 10 \mathrm{NiTi}$ austenitic steel over a wide range of displacement rates, Journal of Nuclear Materials 399(1) (2010) 114-121.

[47] V.V. Bryk, O.V. Borodin, A.S. Kalchenko, V.N. Voyevodin, V. Ageev, A. Nikitina, V. Novikov, V. Inozemtsev, A. Zeman, F.A. Garner, Ion issues on irradiation behavior of structural materials at high doses and gas concentrations, 11th International Topical Meeting on Nuclear Applications of Accelerators, Bruges, Belgium, 2013.

[48] E. Aydogan, T. Chen, J. Gigax, D. Chen, X. Wang, P. Dzhumaev, O. Emelyanova, M. Ganchenkova, B. Kalin, M. Leontiva-Smirnova, R. Valiev, N. Enikeev, M. Abramova, Y. Wu, W. Lo, Y. Yang, M.P. Short, S.A. Maloy, F.A. Garner, L. Shao, Effect of self-ion irradiation on the microstructural changes of alloy EK-181 in annealed and severely deformed conditions, Journal of Nuclear Materials in review. 
Tables and Figures

Table 1. Surface sputtering yield at different ion energies for an ion fluence of $5 \times 10^{17}$ ions $/ \mathrm{cm}^{2}$.

\begin{tabular}{|c|c|c|c|c|c|}
\hline $\begin{array}{c}\text { Ion Energy } \\
(\mathrm{MeV})\end{array}$ & $\begin{array}{c}\text { Peak Dose } \\
(\mathrm{dpa})\end{array}$ & $\begin{array}{c}\text { Average Ion } \\
\text { Range (nm) }\end{array}$ & $\begin{array}{c}\text { Thickness Increase } \\
\text { Due to Injected Ions } \\
(\mathrm{nm})\end{array}$ & $\begin{array}{c}\text { Sputtering Yield } \\
(\text { atoms/ion) }\end{array}$ & $\begin{array}{c}\text { Sputtered } \\
\text { Thickness (nm) }\end{array}$ \\
\hline 0.5 & 770 & 175 & 59 & 3.55 & 209.3 \\
\hline 1.8 & 575 & 617 & 59 & 1.82 & 107.5 \\
\hline 3.5 & 515 & 1072 & 59 & 1.22 & 72 \\
\hline 5.0 & 505 & 1381 & 59 & 0.88 & 52 \\
\hline
\end{tabular}




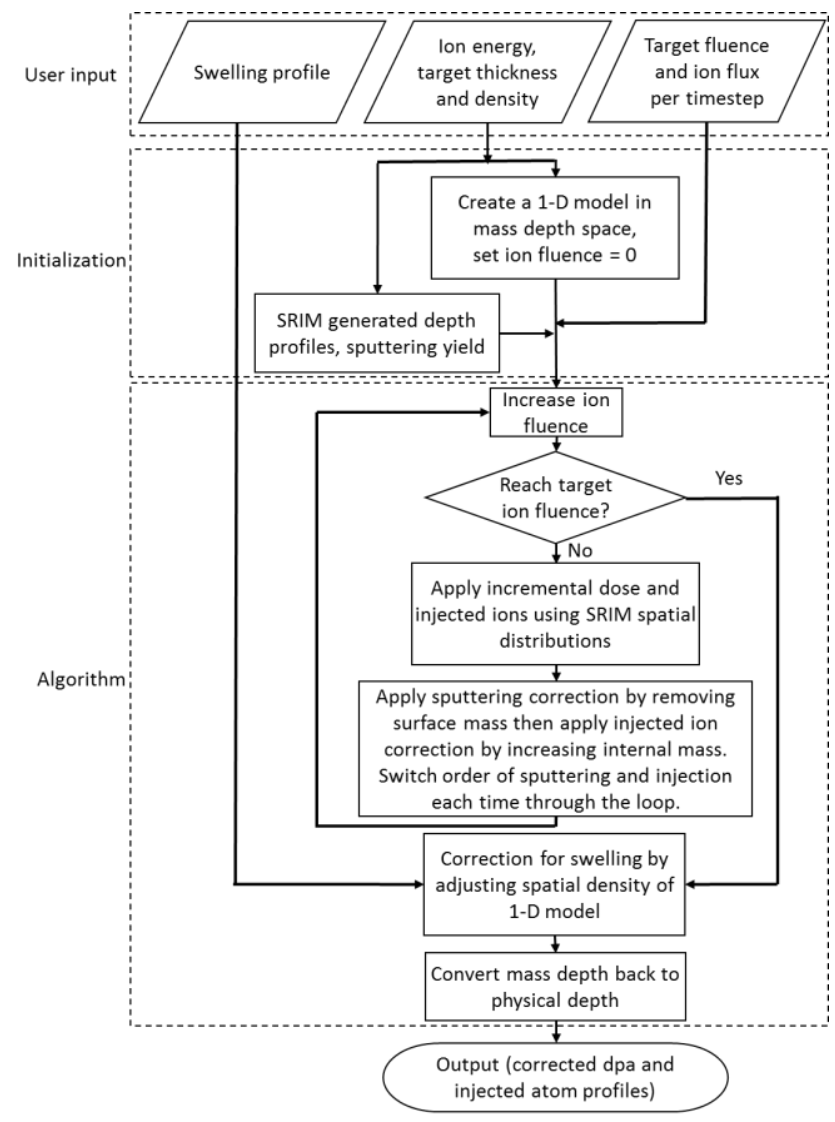

Figure 1. Flow chart of processes for calculating corrected dpa and injected ion profiles.
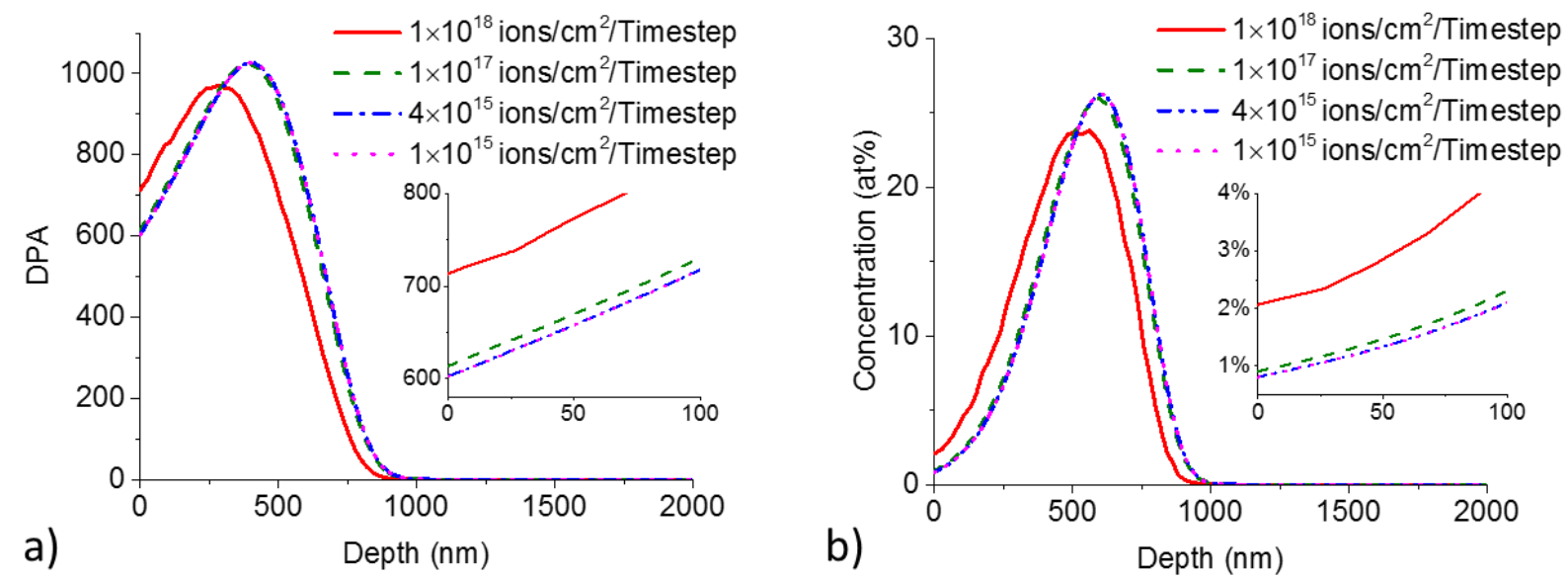

Figure 2. Comparison of a) dose profiles and b) injected ion concentration distributions obtained from various fluence per time step values for a total fluence of $1 \times 10^{18}$ ions $/ \mathrm{cm}^{2}$. The insets on the right of both 
figures are enlarged regions from 0 to $100 \mathrm{~nm}$. A fluence time step of $4 \times 10^{15}$ ions $/ \mathrm{cm}^{2}$ or less was needed to produce invariance in the profiles.
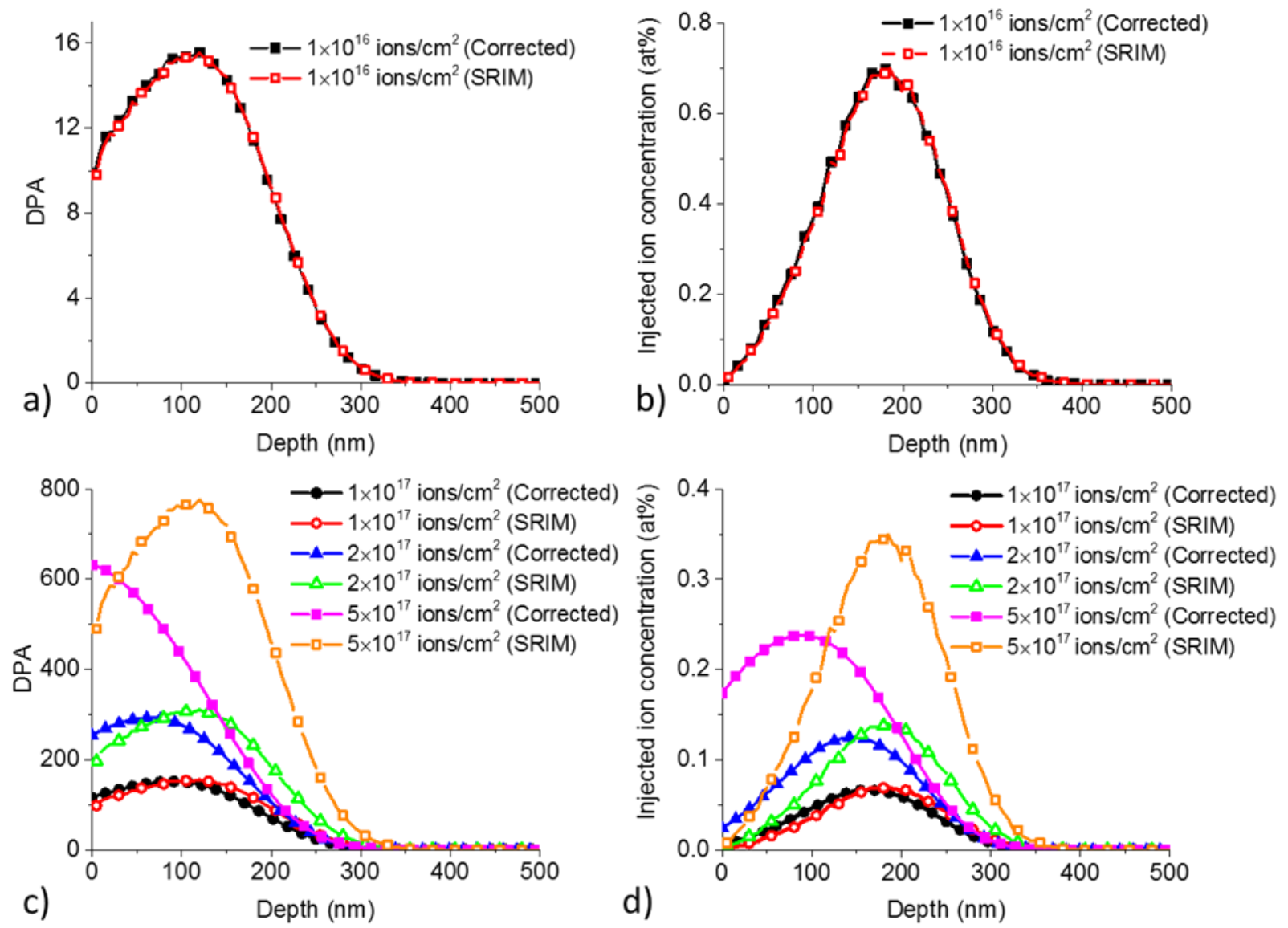

Figure 3. SRIM-calculated (dashed lines) and corrected profiles (solid lines) for: a) and c) dose distribution, and b) and d) injected ion distribution for $500 \mathrm{keV} \mathrm{Fe}$ ion irradiation onto an Fe target at various fluences. At lower ion fluence there is little difference between SRIM and the corrected profiles as shown in figure a) and b). 

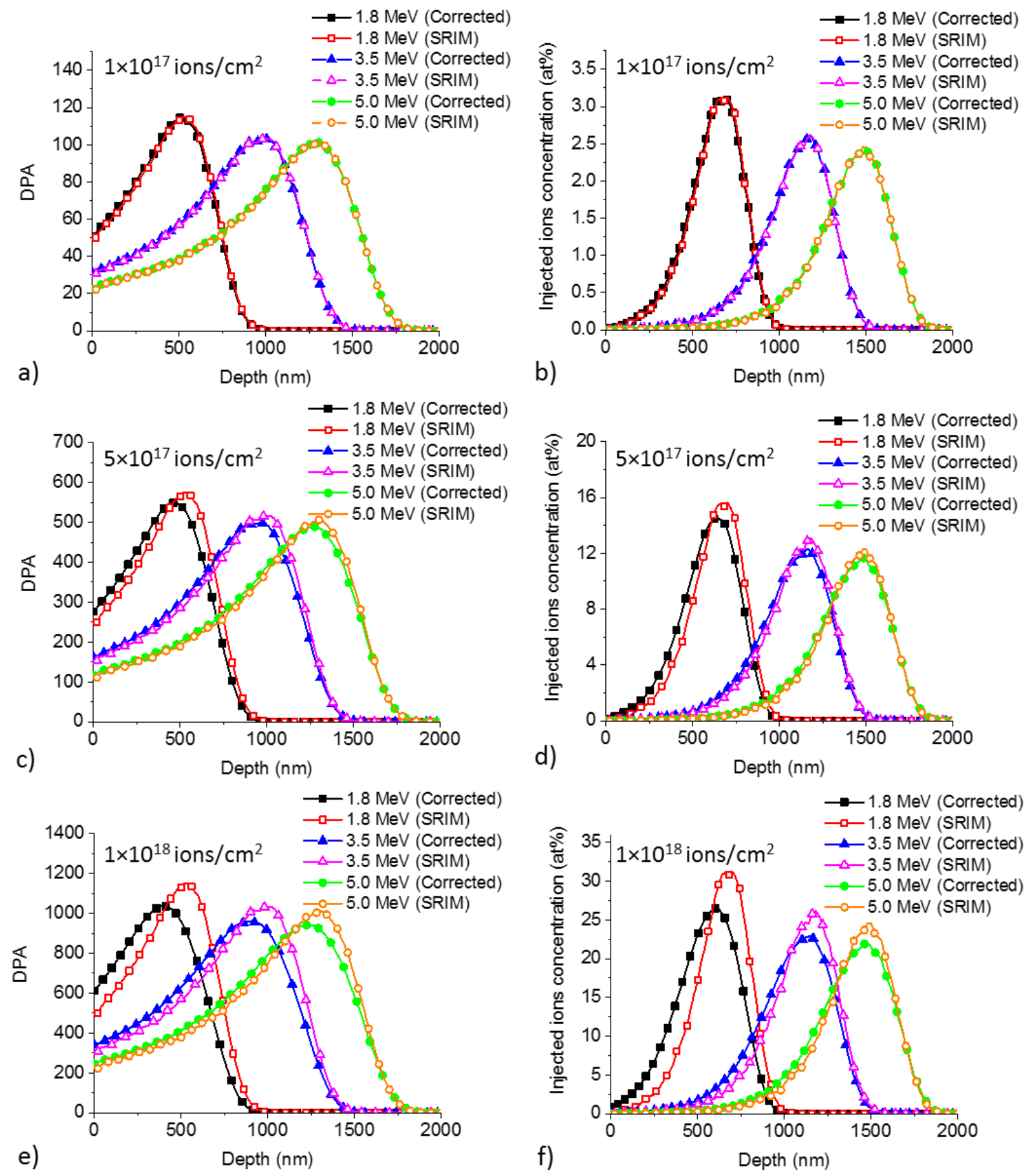

Figure 4. Comparison of SRIM-calculated (dashed lines) and corrected dose and injected ion profiles (solid lines) for energies of $1.8 \mathrm{MeV}, 3.5 \mathrm{MeV}$ and $5.0 \mathrm{MeV}$ at fluence of (a-b) $1 \times 10^{17} \mathrm{ions} / \mathrm{cm}^{2}$, (c-d) $5 \times 10^{17}$ ions $/ \mathrm{cm}^{2}$, and $(\mathrm{e}-\mathrm{f}) 1 \times 10^{18}$ ions $/ \mathrm{cm}^{2}$. 

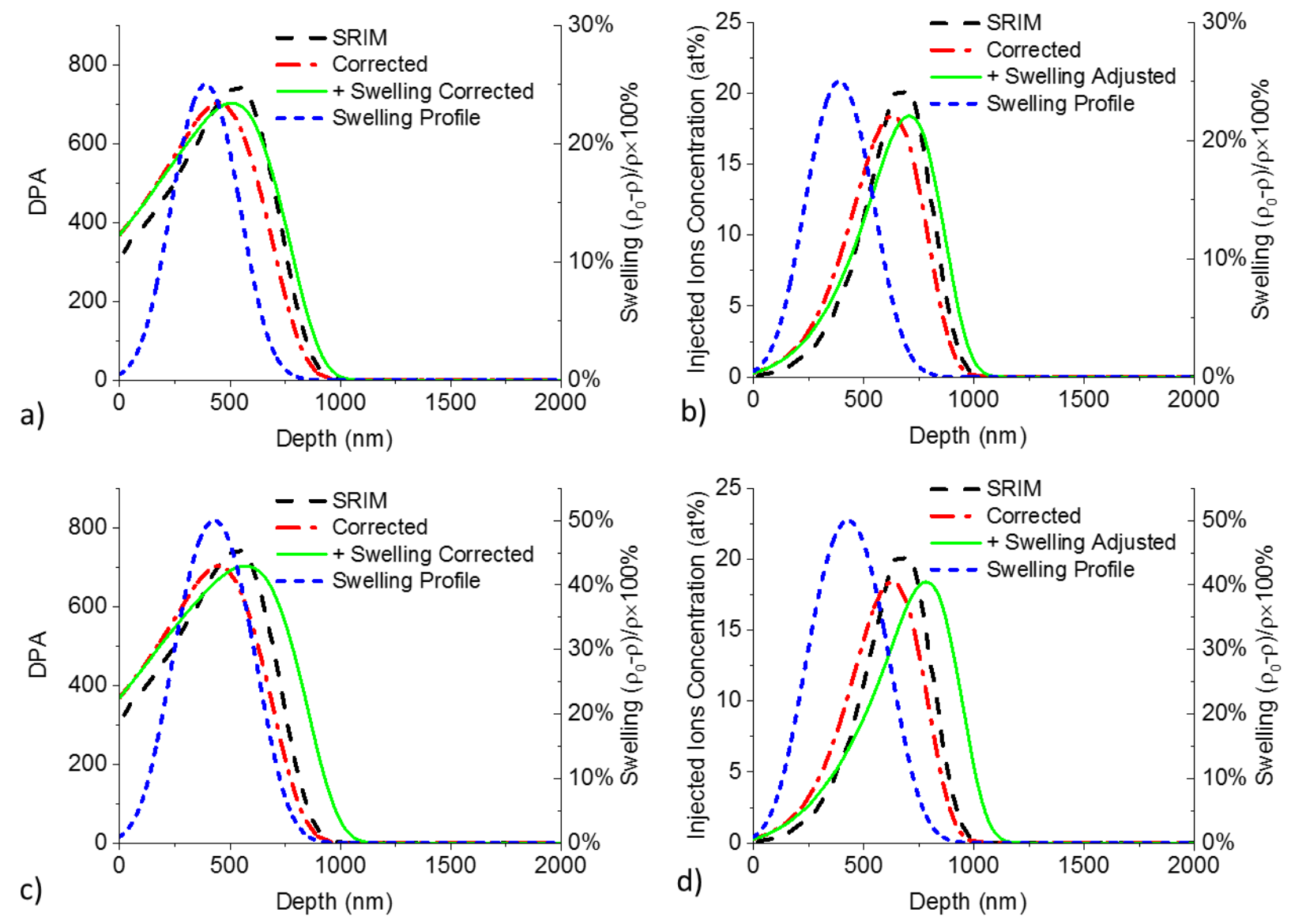

Figure 5. Swelling effects on final dose profiles for a) and c), and injected ion distributions for b) and d) using $1.8 \mathrm{MeV}$ ions with a fluence of $6.5 \times 10^{17}$ ions $/ \mathrm{cm}^{2}$. In a) and b), the peak swelling was assumed to be $25 \%$ while in c) and d) the peak swelling was assumed to be $50 \%$. 

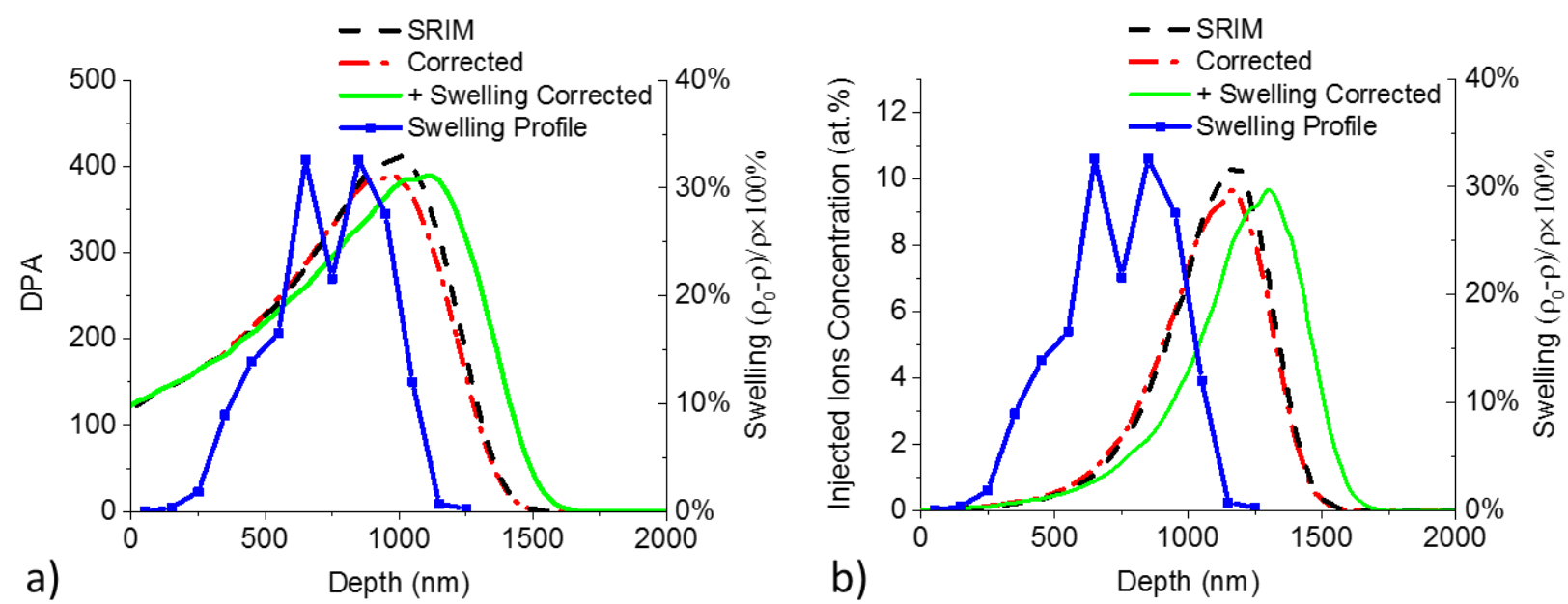

Figure 6. Swelling effects on a) final dose profiles and b) injected ion distributions using $3.5 \mathrm{MeV} \mathrm{Fe}$ ions to peak damage of 400 dpa of EK-181. The swelling profile was adopted from TEM cross-section measurements. 